\title{
Impact Analysis of Hydration and Sleep Intervention Using Regression Techniques
}

\author{
K. Reddy Madhavi ${ }^{\mathrm{a}}$, G.Sunitha ${ }^{\mathrm{b}}$, J.Avanija ${ }^{\mathrm{c}}$, S. Viswanadha Raju ${ }^{\mathrm{d}}$, and Sindhooja Abbagalla ${ }^{\mathrm{e}}$ \\ a \\ Associate Professor, Department of Computer Science and Engineering, Sree \\ Vidyanikethan Engineering College, A.Rangampet, Tirupati \\ bProfessor, Department of Computer Science and Engineering, Sree Vidyanikethan Engineering College, A.Rangampet, \\ Tirupati \\ ${ }^{\mathbf{c}}$ Associate Professor, Department of Computer Science and Engineering, Sree \\ Vidyanikethan Engineering College, A.Rangampet, Tirupati \\ dAssociate Professor, Department of Computer Science and Engineering, JNTUHCEJ, Jagithyal \\ e Department of Computer Science and Engineering, NIT Puducherry
}

Article History: Received: 11 January 2021; Accepted: 27 February 2021; Published online: 5 April 2021

\begin{abstract}
Health is Wealth,"- The most well-known golden maxim, but most of the time, we ignore our health in favor of our professional pursuits. Good health covers not just our physical but also our psychological well-being. Individuals have forgotten that preserving our wellbeing contributes a large amount of effort to our advancement in this growing-race. Individuals agree that juggling the demands of life in clinical medicine poses a unique challenge, but good health can be sustained by drinking plenty of water and sleeping enough. We conducted an online questionnaire survey with a total of 30 questions, and the data gathered will be used to continue our hypotheses. Our main goal in this study will be to focus on the significance of drinking water and sleep respective to their age using machine learning regression algorithms. Therefore, a corrective statistical analysis can be predicted based on a dependent and independent variable.
\end{abstract}

Keywords: Health, drinking water, machine learning, sleep, and regression.

\section{Introduction}

Superior health is not only an absence of illness but a comprehensive of physical, mental, and social wellbeing. In 1986 World Health Organisation (WHO) had also stated that health is a way of empowering individuals and enabling them to lead a life of meaning and purpose [1]. Mental and Physical health are two widely recognized forms of health, although physical health differences can be identified by different measures, mental health diagnosis is far more complicated. Therefore, maintaining good mental care has become very essential.

Due to its core global roles of developing, monitoring, and implementing international norms and standards, as well as coordinating various players against shared objectives, World Health Organization (WHO) plays an important role in global health and disease governance [2]. Since balancing one's wellbeing in the pursuit of development has always been a challenge, healthcare practitioners and organizations are constantly attempting to assist individuals in maintaining their health through efficient and easy tactics.

This research's main objective is to illustrate that age, water and sleep are keys for optimal human performance. Machine Learning Regression algorithms are been used to show the statistical learning for the importance of health. Assessment of the data obtained from the online questionnaire survey will be used to carry on the analysis using simple and multiple linear regression techniques to estimate the correlation between the target and independent variables.

The rest of the paper is organized as follows: Section 2 concentrates on the relevant survey, section 3 focuses on the proposed method, section 4 provides results and discussion and section 5 concludes with future work.

\section{Relevant Survey}

Medical Researches claimed that consuming the right amount of drinking water and having ample sleep respective to their age and gender helps in reducing the risks trapping towards health issues. The attributes age, water, and sleep are not independent variables but are also interrelated to each other [3]. As we know human body consists of $60 \%$ of water and multiple activities are carried out continuously in the human body and water is the only essential ingredient that contributes to work smoothly and maintain a resolute body temperature [4]. Human body hydration has always been beneficial by physical and mental effects but on the other hand, dehydration often has severe effects on the human body [5]. Sleep deprivation is the most crucial effect which tends to lead to dehydration [6].

Satisfactory sleep plays a key role in the regulation of the brain and systematic functioning across the body. Sleep deprivation is usually referred to as sleep disturbances. Sleep disorder has opened to severe diseases such as increases incidences of cardiovascular morbidity, diabetics, blood pressure, kidney disorders, obesity, and many more [7]. New Researches claim that suffering from sleep deprivation may be triggered due to dehydration of our body by potentially disrupting the hormonal releases [8]. According to the study of the institute of medical 
a human body have to consume a minimum of 2.2litres of drinking water for women's and 3.2 liters of water for men greater than or equal to 18years of age to keep their body hydrated.

\section{Proposed Method}

\subsection{Dataset used}

An online self-administered questionnaire survey was designed consisting of socio-demographic questions followed by questions about the changes that occurred in their self-routine mainly considering their health and work owing to the current pandemic. The questionnaire comprised a total number of 30 questions that a total of 401 people responded. The data provided by the respondents is used as the dataset in this paper. In this paper, we worked on some attributes such as consumption of water in liters, the amount of time spent sleeping at night in hours, and the health issues one faces. Our questions related to daily drinking water intake included options in the form of range values such as <1litres, 1-2litres, 2-3litres, 3-4litres, and >4litres. Similarly, we have provided range values for sleeping hours that include options such as $<6$ hours, 6-8hours, and mostly disturbed sleep. Our other attribute on health issues one is facing and it is very worthy to remember that $81.5 \%$ of our respondents did have not any health issues. This questionnaire data has been divided based on their gender which provided us that a total of 239 males and 164 females had been contributed to this questionnaire. Below are the screenshots of questions from the questionnaire required to extract the attributes data.

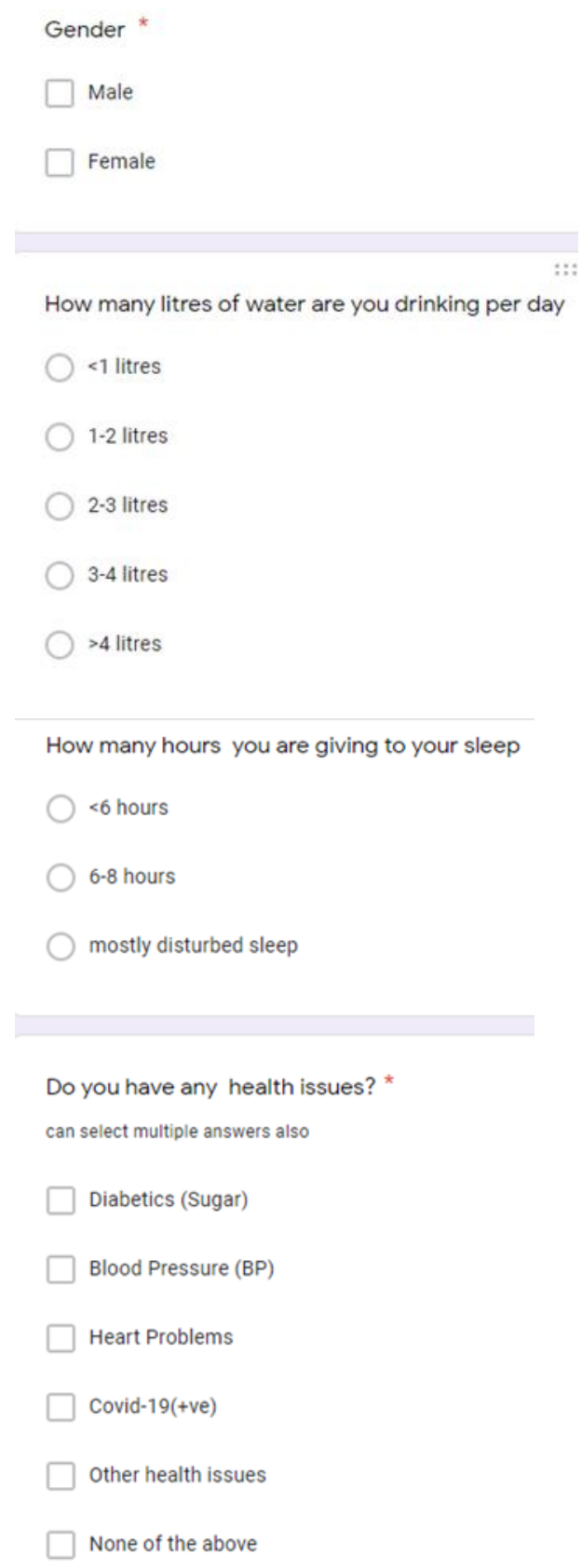

\subsection{Importance of target and independent variables}

The analysis research was converged on both the dependent and the independent factors considered. In the study, the dependent variables were recognized as criteria for health. The value of dependent variables fluctuates 
with the values of independent variables in the regression analysis. In simple linear regression, variable drinking water is considered dependent on the independent variable age. Similarly, considering another set of attribute's sleep is known to be dependent on the independent variable drinking water. Multiple linear regression indicates that the dependent variable health is correlated with independent variables- age, drinking water, and sleep. Drinking water and sleep attributes were presented in the questionnaire with range values of which their upper values were used for our ease. Evaluating the health attribute 0 is no health issue and 1 is having some health issue.

\section{Regression Method}

Regression models for machine learning such as simple linear regression and multiple linear regression are been used here. Root Mean Squared Error (RMSE) is used to estimate the accuracy of the predictions.

\subsection{Simple Linear Regression}

Linear regression measures correlation between the dependent an independent variables. It is a simulation technique where a dependent attribute is predicted based on independent variables. Based on the statistical analysis linear regression equation obtained will be in the following form,

$$
\mathrm{y}=\mathrm{mx}+\mathrm{c}
$$

where $\mathrm{y}$ is the dependent variable, $\mathrm{x}$ is the independent variable and c represents constant [9].

Applying this linear regression on our dataset and splitting the attributes into two sets in which the first set includes attributes such as age and drinking water and the second set includes attributes such as drinking water and sleep. This simulation model illustrates age as an independent variable and drinking water as the dependent variable. As age increases intake of drinking water may or may not increase. Therefore, by analyzing the data the regression equation for drinking water in males indicating RMSE value as 0.8625 is as follows,

$\mathrm{y}=0.0139 * \mathrm{x}+2.6599$

where $\mathrm{y}$ indicates intake of drinking water in liters and $\mathrm{x}$ indicates Age in years.

Similarly, analyzing the equation for drinking water in females that indicates RMSE 0.9417 is as follows:

$$
y=0.0174 * x+2.3702
$$

where $y$ indicates intake of drinking water in liters and $\mathrm{x}$ indicates age in years.

Applying the above technique on another set of attributes such as sleeping hours and intake of drinking water where sleep is a dependent variable and intake of drinking water is considered as the independent variable. Correspondingly, our sleep mostly depends on the consumption of water. By analyzing the data, the regression equation for predicting how much one can sleep peacefully on amount of drinking water in a day in males indicates RMSE value such as 0.3990 is as follows,

$$
y=-0.0032 * x+7.6533
$$

where $\mathrm{y}$ indicates sleeping hours and $\mathrm{x}$ indicates intake of drinking water in liters.

Similarly, analyzing the equation regression equation for females indicating RMSE value 0.5287 is as follows,

$$
y=-0.0052 * x+7.3654
$$

where $\mathrm{y}$ indicates sleeping hours and $\mathrm{x}$ indicates intake of drinking water in liters.

\subsection{Multiple Linear Regression}

Multiple Linear Regression helps us to find a relationship between one dependent variable and two or more independent variables. Based on the statistical analysis multiple linear regression model provides equation such as,

$$
y=m_{1} x_{1}+m_{2} x_{2}+\cdots m_{n} x_{n}+c+\varepsilon
$$

where $x_{1}, x_{2 \ldots}, x_{n}$ represents independent variables, y represents the dependent variable, $\varepsilon$ means error value and $\mathrm{c}$ represents the constant [10].

This modeling technique when applied to our dataset provides us relation health depends on independent variables such as age, intake of drinking water, and time spent on our sleep. Based on the statistical analysis the regression equation obtained for males indicates RMSE value 0.3573 is as follows,

$$
y=0.0069 x_{1}-0.0122 x_{2}-0.0774+0.5512
$$

where y indicates health issue, $x_{1}$ indicates age, $x_{2}$ indicates intake of drinking water in liters and $x_{3}$ indicates how many hours one spends on sleep.

Similarly, analyzing the equation regression equation for females indicates RMSE value 0.3926 is as follows,

$$
y=0.0009 x_{1}-0.0415 x_{2}-0.0202 x_{3}+0.20714
$$


where y indicates health issue, $x_{1}$ indicates age, $x_{2}$ indicates intake of drinking water in liters and $x_{3}$ indicates how many hours one spends on sleep.

\section{Results and Discussion}

The data collected here covers people 17-65 years of age therefore our study is only applicable to people 17 years of age and older. Our dataset included 401 responses out of which 239 males and 164 females. Based on our dataset we have developed our regression models such that it would help us to predict the minimum amount of water intake that is related to get ample sleep and which helps us to maintain good health. According to the World Health Organization, individuals must consume a minimum of 2litres of water and this intake of water differs by health effects and environment.

Our proposed model works well in the Indian scenario, but it would need to be changed if our environments differed. As a result, different environmental datasets will result in different performances.Fig: 1 demonstrates the actual versus predicted values for the intake of drinking water for males and females. Similarly, fig:2 and fig:3 plots actual and predicted values relate to sleep associating with the intake of drinking water and health is dependent on attributes such as age, intake of drinking water, sleep.

According to our study, the minimum amount of water one must consume every day to keep their body hydrated is related to their gender and age. It is being observed that equation 1is being predicting the minimum amount of intake of water for an individual based on their age. But the visualizing fig: 1 it is showing lower predicted values for higher actual values, which may be occurring because our consumption of water also depends on various other variables, which demand that we inevitably drink more water which is beneficial and needed for our health.

Similarly, it is important to have 8 hours of sleep depending on your age but there are some individuals facing problems such as sleep adequately. Researchers have concluded that sleep deprivation can be caused due to dehydration due to inadequate drinking water consumption, which affects our physical and mental health. Based on their consumption of water, equation 2 is determining the amount of sleep an individual can have. This is resulting that one can sleep approximately 7 hours even for those who consume too little drinking water. This may be because human body can indeed absorb water from food. Therefore, more study needs to be conducted on it is only drinking water that affects our sleep or even our diet and other liquids affects our sleep too.

Envisioning the analysis provides a framework to ensure that our consumption of drinking water and ample sleep decreases our risk of being unhealthy. The study reveals the predicted values individuals classify that people consuming the right amount of drinking water and having ample sleep their risk of being affected by any health issues is less than or equal to 0.5 than people who cannot have enough sleep. There are some exceptions to our study that will be better listed in future studies.

The strengths of this study are that it allows us to provide a remedial statistical overview of our health and that is dependent on the intake of drinking water, good sleep according to their age will help us improve our health which associate both physically and mentally.

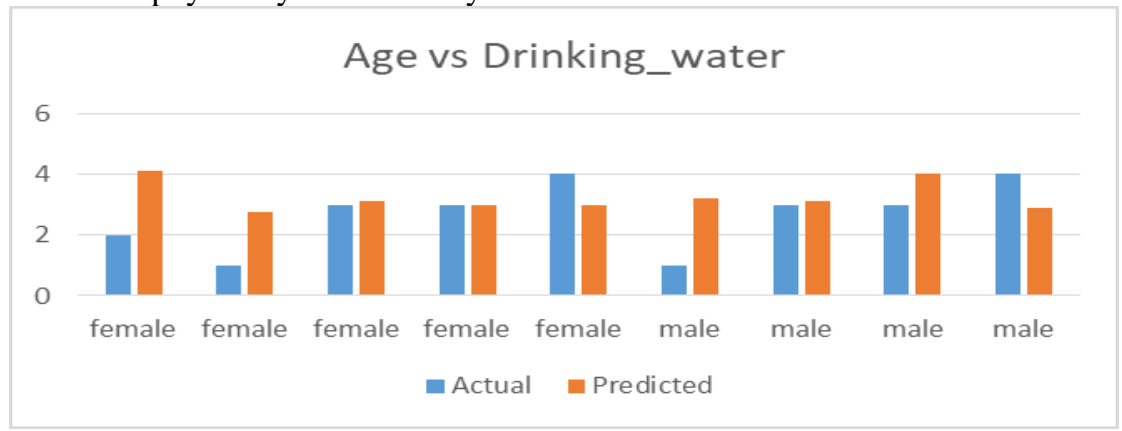

Fig.1 Plot bar graph of actual versus predicted values for the intake of drinking water

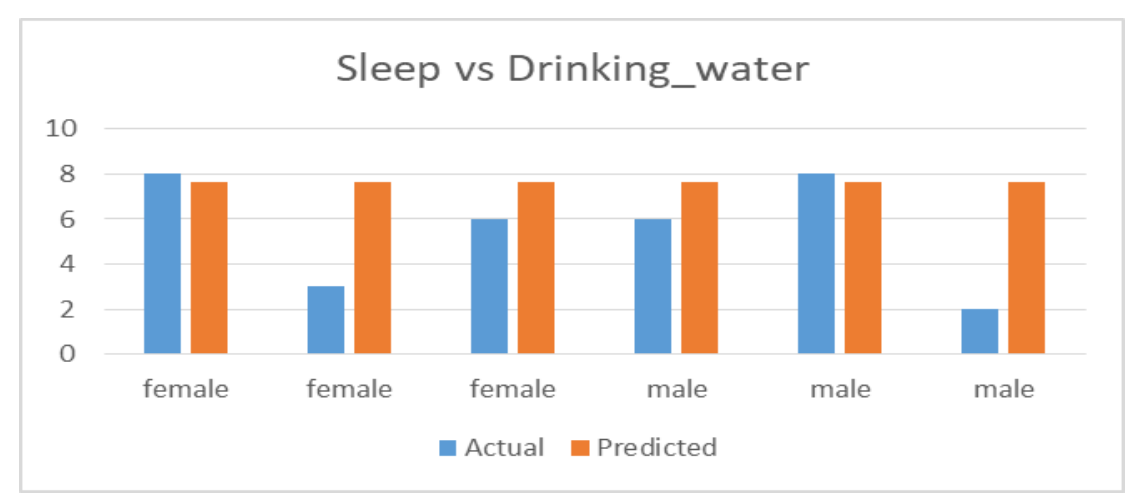

Fig.2 Plot bar graph of actual versus predicted values minimum time spent on sleep 
Fig.2 Plot bar graph of actual versus predicted values

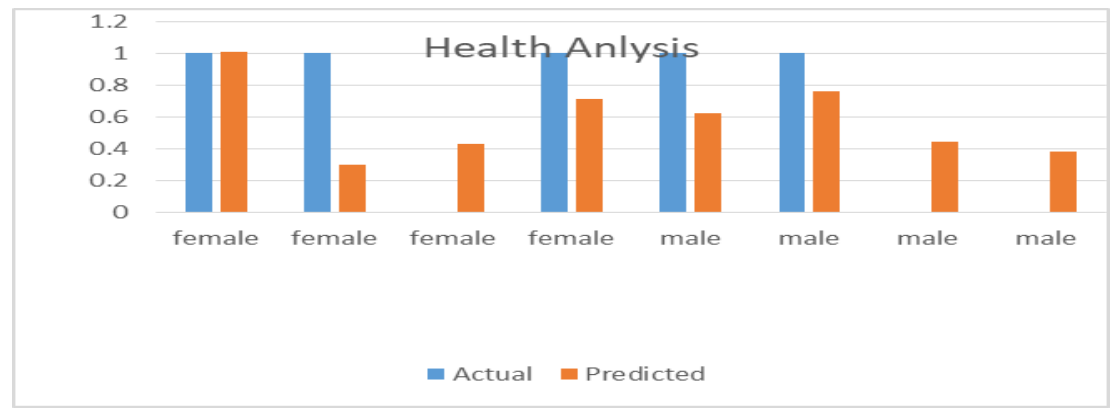

Fig.3 Plot bar graph of actual versus predicted value for health analysis

\section{Conclusion}

The main aim was to provide the basic analysis on consuming the right amount of drinking water and having ample sleep contribute to a tremendous effect on our health. Using machine learning regression algorithms $\backslash$, we evaluated the correlation between water and sleep by age and gender respectively. It also offers statistical analysis for study our health statistics. Further studies are required to measure other attributes obtained through the online survey and their association with our health effects and also to implement a different model that works on different environmental datasets resulting in different performances.

\section{References}

1. Adam Felman (19 April 2020) "What is Good Health".

2. Ruger, Jennifer Prah, and Derek Yach. "The Global Role of the World Health Organization." Global health governance: the scholarly journal for the new health security paradigm vol. 2,2 (2009): 1-11.

3. Guest C., Guest D.D., Smith-Coggins R. (2020) How to Care for the Basics: Sleep, Nutrition, Exercise, and Health. In: Roberts L. (eds) Roberts Academic Medicine Handbook. Springer, Cham.

4. Ashley Marcin(March 7, 2019)," How much water one should drink"

5. Chard AN, Trinies V, Edmonds CJ, Sogore A, Freeman MC (2019) The impact of water consumption on hydration and cognition among schoolchildren: Methods and results from a crossover trial in rural Mali. PLoS ONE 14(1): e0210568. https://doi.org/10.1371/journal.pone.0210568

6. Ana Sandoiu (November 7, 2018), "Sleep deprivation caused by dehydration".

7. Chattu, V.K.; Manzar, M.D.; Kumary, S.; Burman, D.; Spence, D.W.; Pandi-Perumal, S.R. The Global Problem of Insufficient Sleep and Its Serious Public Health Implications. Healthcare 2019, 7, 1

8. Dr. Michael Breus (Feb 17, 2019)," The Relationship between water and sleep is a two-way street- How to avoid Dehydration".

9. Raju, M. S. "Unit-16 Simple Linear Regression." IGNOU, 2020.

10. JOUR, Kaya Uyanık, Gülden, Güler, Neşe," A Study on Multiple Linear Regression Analysis" (2013) Procedia - Social and Behavioral Sciences 